\title{
-Original一
}

\section{Identification of Ryu's Segmentation of the Liver Using MDCT Analysis}

\author{
Takahisa Kaneko', Takeshi Tomiyama ${ }^{1}$, Hajime Kiyuna ${ }^{1}$, \\ Tadashi Machida' ${ }^{2}$ Hiromitsu Hayashi ${ }^{2}$ and Shin-ichiro Kumita ${ }^{2}$ \\ 'Sixth-year Student, Medical Department, Nippon Medical School \\ ${ }^{2}$ Department of Clinical Radiology, Graduate School of Medicine, Nippon Medical School
}

\begin{abstract}
Background: Couinaud's segmentation is widely used for clinical segmentation of the liver. Recently, a new method of liver segmentation was described by Ryu. In this method, the right posterior sector in Couinaud's segmentation is regarded as 1 segment, and the right anterior sector is subdivided into the antero-ventral segment (AVS) and the antero-dorsal segment (ADS), which are demarcated by the anterior fissure vein (AFV). We used data from multi-detector row computed tomography (MDCT) to identify the portal and hepatic veins that are used as anatomical landmarks in both Couinaud's segmentation and Ryu's segmentation.

Methods: We analyzed data from 100 patients who had no space occupying lesions of the liver and had undergone abdominal CT examination from June through September 2008. MDCT was used to obtain contrast-enhanced helical scans of the whole liver during the portal venous phase. Using thin-slice data, we attempted to identify the portal and hepatic veins, in particular the anterior fissure vein (AFV), the antero-ventral portal vein (AVPV), and the antero-dorsal portal vein (ADPV), all of which are used in Ryu's segmentation.

Results: In all cases, we were able to identify the hepatic segments of Couinaud's segmentation. However, in several cases, we were unable to identify the segments used in Ryu's segmentation. The AFV flowed into the intermediate hepatic vein (IHV) in 88\% of cases and into the right hepatic vein (RHV) in $12 \%$. Among the former, the AFV flowed into the proximal IHV in $53 \%$ of cases. Although the AFV could be identified in these cases, it was difficult to determine the border between the AVS and the ADS.

Conclusions: Our findings indicate that the use of MDCT data to identify hepatic anatomy was more difficult in Ryu's segmentation than in Couinaud's segmentation, because the AFV could not be easily identified in the former. Indeed, even when the AFV could be identified, its use as a landmark was often questionable.

(J Nippon Med Sch 2010; 77: 244-249)
\end{abstract}

Key words: liver segmentation, Couinaud's segmentation, Ryu's segmentation, multi-detector row CT $(\mathrm{MDCT})$

Correspondence to Tadashi Machida, Department of Radiology, Nippon Medical School, 1-1-5 Sendagi, Bunkyo-ku, Tokyo 113-8603, Japan

E-mail: zeke_atom@yahoo.co.jp

Journal Website (http://www.nms.ac.jp/jnms/) 


\section{Introduction}

Couinaud's segmentation ${ }^{1}$ is widely used for clinical segmentation of the liver. It is a functional segmentation based on the location of vessels and biliary ducts. However, the boundaries between segments are not always clear. For example, segments V and VIII and segments VI and VII are not divided by anatomical landmarks but rather by an imaginary plane named the transverse scissura ${ }^{2}$, transverse portal $\operatorname{arch}^{3}$, or main portal $\operatorname{arch}^{4,5}$. Therefore, several new methods of segmentation have been proposed, including one developed by $\mathrm{Ryu}^{4}$, which divides the liver into 7 segments based on the positions of portal and hepatic veins. Ryu maintains that this method of segmentation has a clearer physiological basis than that of Couinaud.

The segmentation of the left lobe (segments II, III, and IV) is identical in Couinaud's and Ryu's segmentations. In Couinaud's segmentation, the right lobe's anterior sector, which is divided into segments $\mathrm{V}$ and VIII, and posterior sector, which is divided into segments VI and VII, are divided by the transverse scissura. In Ryu's segmentation, the posterior sector is regarded as 1 segment, and the anterior sector is divided into the antero-ventral segment (AVS) and the antero-dorsal segment (ADS). Ryu reported that the anterior fissure vein (AFV), which is useful as a landmark, traverses the boundary between the AVS and ADS and joins the intermediate hepatic vein (IHV) near the junction of the IHV and inferior vena cava (Fig. 1).

We used multilayer data from multi-detector row computed tomography (MDCT) to determine whether anatomical imaging analysis based on Ryu's segmentation was possible. To do so, we created 3dimensional images by tracing and identifying portal and hepatic veins. In addition, we used these images to describe the anatomical features of the AFV, which have been described in only a few studies.

\section{Materials and Methods}

We reviewed the records of 100 patients (mean age: 61.9 years; range: $4-87 ; 58$ male patients and 42 female patients) who underwent abdominal CT examination from June through September 2008. Patients with space occupying lesions of the liver were excluded from the study.

A 64- or 32-row multidetector-row CT scanner (LightSpeed VCT, LightSpeed VCT Select, GE Healthcare, Tokyo, Japan) and a workstation (Advantage Windows Version 4.3, GE Healthcare, Tokyo, Japan) were used. The scanning parameters were collimation 0.625 or $1.25 \mathrm{~mm}$. Contrastenhanced helical scans of the whole liver during the portal venous phase were obtained. As a contrast agent, $150 \mathrm{~mL}$ of iohexol 300 (Omnipaque 300; Daiichi Sankyo, Tokyo, Japan) was injected at a rate of 3 $\mathrm{mL} / \mathrm{sec}$ with an automated injector in 5 adult patients, whose CT data were reconstructed into three-dimensional images. In 94 adult patients, 100 $\mathrm{mL}$ of iohexol 300 was injected similarly. In 1 infant patient, $2 \mathrm{~mL} / \mathrm{kg}$ of iohexol 300 was injected at a rate of $2 \mathrm{~mL} / \mathrm{sec}$ with an automated injector. The volume data were used in the subsequent analysis. From CT volume data of 5 randomly chosen cases, axial images $(0.625-\mathrm{mm}$ collimation with $0.625-\mathrm{mm}$ reconstruction interval) were reconstructed. From these images, the portal veins, hepatic veins, and hepatic segments were identified based on Couinaud's segmentation. Then, the antero-ventral portal vein (AVPV), the antero-dorsal portal vein (ADPV), and the AFV were identified based on Ryu's segmentation and color coded.

Using the data of these 5 cases and abdominal contrast-enhanced CT data (5-mm collimation with 5$\mathrm{mm}$ reconstruction interval axial image) from the 95 cases, the hepatic and portal veins were identified.

We then examined whether classification based on Couinaud's segmentation was possible, and whether the AVPV, ADPV, and AFV specified in Ryu's segmentation could be identified. We defined the $\mathrm{AFV}$ as a vein located between the AVPV and ADPV and flowing into the IHV or right hepatic vein (RHV). We also attempted to identify the point of drainage for the AFV. The point where the AFV flowed into the IHV or RHV was classified as proximal, medial, or distal. The proximal position was subdivided into halves (Fig. 2). 


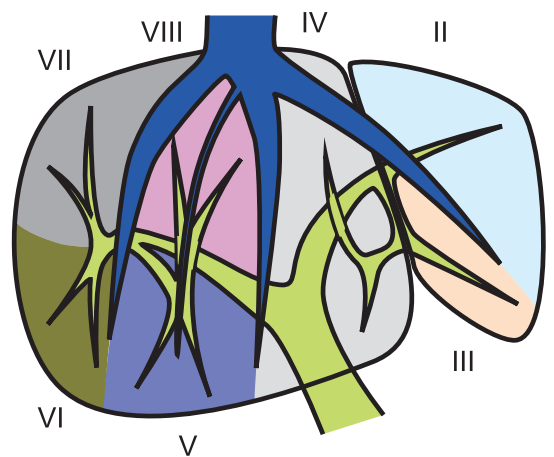

(a)

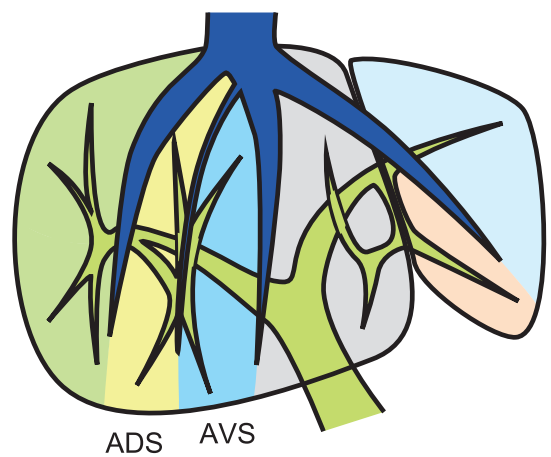

(b)

Fig. 1 Comparison of Couinaud's segmentation and Ryu's segmentation. (a) Couinaud's segmentation, (b) Ryu's segmentation

In the right lobe of Couinaud's segmentation, the anterior sector is divided into segments V and VIII, and the posterior sector is divided into segments VI and VII. In Ryu's segmentation, the posterior sector is regarded as 1 segment, and the anterior sector is divided into the AVS and ADS.

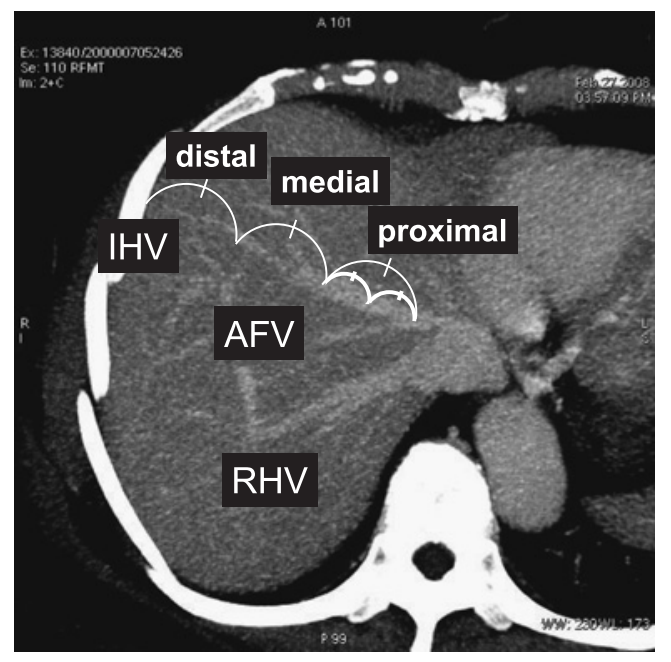

Fig. 2 Drainage of the AFV (Slab MIP, axial)

The point at which the AFV flows into the IHV was classified as proximal, medial, or distal. Furthermore, the proximal region was divided into 2 halves.

In this image, the AFV flows into the proximal IHV. IHV: intermediate hepatic vein RHV: right hepatic vein

\section{Results}

\section{Underlying Disease}

Details of underlying disease are shown in Table 1. There were 20 fatty liver patients. Regardless of the underlying disease of the patients, we were able to identify the vessels as follows.

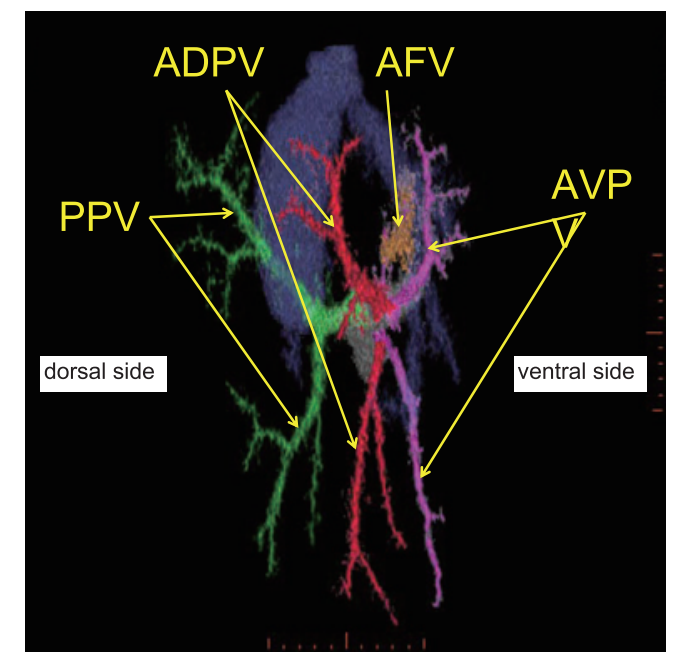

Fig. 3 Three-dimensional right anterior view of Ryu's segmentation (volume rendering); the $\mathrm{AFV}$ can be seen between the AVPV and ADPV.

PPV: posterior portal vein ADPV: antero-dorsal portal vein AVPV: antero-ventral portal vein

Identification in Couinaud's and Ryu's Segmentation

In all 5 images examined, we were able to identify all segmental branches of the portal vein, as required in Couinaud's and Ryu's methods of segmentation. The AFV between the AVS and ADS is shown in Figure 3.

\section{Identification of AVPV and ADPV}

In all cases, we were able to identify the hepatic 
MDCT Analysis of Ryu's Segmentation

Table 1 Underlying disease in 100 patients

\begin{tabular}{|c|c|c|}
\hline \multirow{13}{*}{ alimentary system } & chronic hepatitis & 14 \\
\hline & drug-indeced hepatitis & 1 \\
\hline & carcinoma of alimentary tract & 33 \\
\hline & biliary tract cancer & 2 \\
\hline & pancreatic neoplastic cyst & 2 \\
\hline & acute pancreatitis & 3 \\
\hline & bile duct stone & 3 \\
\hline & cholecystitis & 1 \\
\hline & appendicitis & 2 \\
\hline & primary biliary cirrhosis & 1 \\
\hline & ileus & 2 \\
\hline & inguinal hernia & 1 \\
\hline & AVM of pancreas & 1 \\
\hline cardiovascular sytem & vena cava inferior obstruction & 1 \\
\hline \multirow{2}{*}{ respiratory system } & pulmonary carcinoma & 5 \\
\hline & pneumothorax & 1 \\
\hline \multirow{2}{*}{ endocrine system } & adrenocortical adenoma & 1 \\
\hline & neuroblastoma & 1 \\
\hline metabolic system & diabetes mellitus & 1 \\
\hline \multirow{2}{*}{ hematopoietic system } & malignant lymphoma & 4 \\
\hline & leukemia & 1 \\
\hline \multirow{5}{*}{ genital system } & ovarian cancer & 5 \\
\hline & uterine cervical cancer & 5 \\
\hline & uterine cervical cancer & 3 \\
\hline & hysteromyoma & 1 \\
\hline & pyosalpinx & 1 \\
\hline mamma & breast cancer & 2 \\
\hline urinary system & pyelonephritis & 1 \\
\hline \multirow[t]{2}{*}{ normal } & normal & 1 \\
\hline & & 100 \\
\hline
\end{tabular}

segments of Couinaud's segmentation. However, we could not identify the AVPV and ADPV, as defined in Ryu's segmentation, in $1 \%(1 / 100)$ of cranial images and 12\% (12/100) of caudal images (Fig. 4).

\section{Identification of the AFV}

Hepatic veins were identifiable in all 100 cases, and the AFV could be identified in $85 \%(85 / 100)$. However, the entire length of the AFV was identifiable across the transverse scissura toward the caudal side in only 1 case.

\section{Vascular Course and Localization of the AFV}

Among the 85 cases in which the AFV was identified, the AFV flowed into the IHV in $88 \%(75 /$ $85)$, and into the RHV in $12 \%(10 / 85)$. Among the former cases, the AFV flowed into the proximal half of the proximal IHV, that is, nearest to the drainage of the IHV, in $53 \%(45 / 85)$ of cases. In $5 \%(4 / 85)$ of the latter, the AFV flowed into the proximal half of the proximal RHV; there were no cases in which the AFV flowed into the distal RHV (Fig. 4). In several cases, the AFV traversed from the cranial to the caudal side. Although the AFV was identifiable in these cases, it was difficult to determine the border between the AVS and ADS.

\section{Discussion}

Regardless of the underlying disease of the patients, we were able to identify the vessels. In the patients with fatty liver, it was easy to identify the vessels, leading is to conclude that the decrease in liver density made the contrast between the liver 


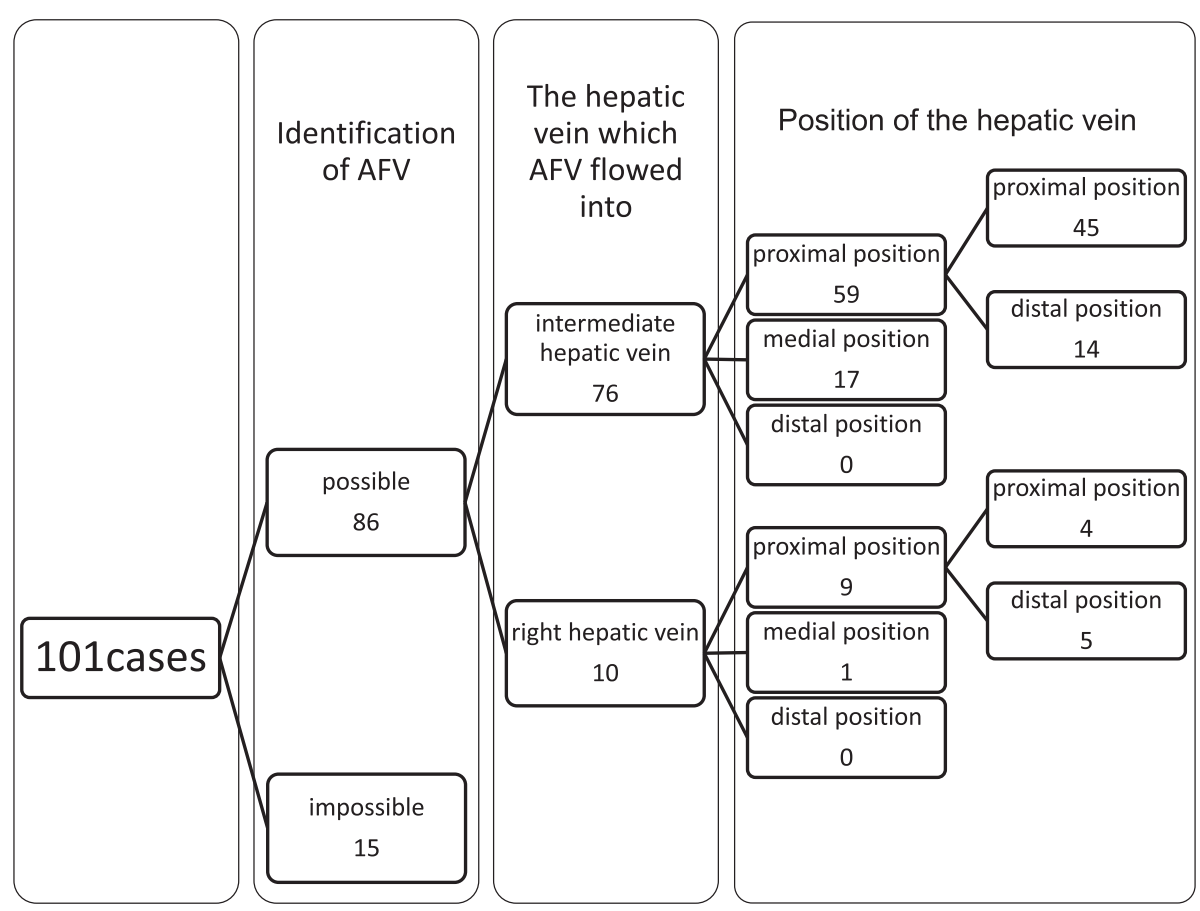

Fig. 4 The regions of the hepatic veins into which the AFV flowed

Table 2 Identification of the AVPV, ADPV, and $\mathrm{AFV}$

\begin{tabular}{lrr}
\hline 101 cases & $\begin{array}{c}\text { Possible to } \\
\text { identify }\end{array}$ & $\begin{array}{c}\text { Impossible to } \\
\text { identify }\end{array}$ \\
\hline Cranial side & $100(99 \%)$ & $1(1 \%)$ \\
Caudal side & $88(87 \%)$ & $13(13 \%)$ \\
AFV & $86(85 \%)$ & $15(15 \%)$ \\
\hline
\end{tabular}

and the liver vessels clear. In the patients with hepatitis, although the liver density was inhomogeneous, we could still identify the vessels.

Shioyama ${ }^{5}$ compared Couinaud's segmentation and Ryu's segmentation using CT data from patients with no liver abnormalities. He reported that there was no difference between them with respect to the difficulty of classifying the segments. However, his understanding of P5 (the portal vein that flows to Couinaud's segment V) differs from ours. Ryu proposed that the region perfused by the third branch ramifying toward the abdominal side is the AVS, and that the region on the dorsal side is the ADS. Shioyama presumed that all portal branches ramifying the area of P5 were AVPV. In the present research, we defined branches ramifying toward the abdominal side in Couinaud's segment $\mathrm{V}$ as AVPV, and those ramifying toward the dorsal side as
ADPV.

Ryu reported that most AFVs flowed into the IHV near the latter's drainage ${ }^{4}$. Our research confirms this: the AFV did indeed flow into a point near the drainage of the IHV in $53 \%(45 / 85)$ of our cases.

We found that the use of MDCT data to classify the AVS and ADS according to Ryu's segmentation was difficult. First, to identify the AFV it is necessary to locate the AVPV and ADPV. Therefore, identification of the AFV was not as straightforward as with other anatomical landmarks, such as the RHV, IHV, left hepatic vein, and the umbilical fissure. Second, in $13 \%(13 / 100)$ of cases, it was impossible to conclusively identify the AVPV and ADPV, which made it impossible to draw precise segment boundaries. Third, the AFV could not be identified in $15 \%(15 / 100)$ of cases. Moreover, the AFV could be identified from Couinaud's segment VIII to segment V in only 1 (1\%) case. In fact, except for this 1 case, the boundary between the AVS and ADS could not be determined, even after identifying the AFV. Finally, in some cases the peripheries of the AFV were in positions superior to the medial AFV. Thus, because of branching and anatomical variation in the $\mathrm{AFV}$, we were able to 
precisely identify the border between the AVS and ADS in only a few cases, which indicates that the AFV lacks universality as an anatomical landmark.

In $15 \%(15 / 100)$ of cases, we were unable to determine the anatomy of the vessels used in Ryu's segmentation. We suspect that inadequate contrast enhancement on radiography was responsible. Increasing the concentration or amount of contrast agent might yield better images of the AFV.

\section{Conclusion}

Because of the difficulty in identifying the AFV using data from MDCT, it was more difficult to identify the hepatic segments of Ryu's segmentation than those of Couinaud's segmentation. Indeed, even when the AFV was identified, it was an inadequate anatomical landmark in many cases.

\section{References}

1. Couinaud C: Lobes et segments hepatiques; Notes sur l'architecture anatomique et chirurgicale du foie. La Presse Medicale 1954; 62: 709-712.

2. Strunk H, Stuckmann G, Textor J, Willinek W: Limitations and pitfalls of Couinaud's segmentation of the liver in transaxial Imaging. European Radiology 2003; 13: 2472-2482.

3. Couinaud C: Dorsal sector of the liver. Chirugie 1998; 123: 8-15.

4. Ryu M, Cho A: In Kanzou No Geka KaibouMonmyaku segmentation $\mathrm{Ni}$ Motoduku Aratana Kankuiki No Kangaekata (Surgical Anatomy of the Liver-New Liver Segmentation Based on Portal Segmentation), 2004; pp 1-6, 25-26, Igaku-Shoin, Tokyo.

5. Shioyama Y, Ikeda H, Sato M, et al.: Couinaud's clasification vs Cho's classification: Their Feasibility in the Right Hepatic Lobe. Japanese Journal of Medical Imaging 2007; 26: 172-179.

(Received, February 22, 2010)

(Accepted, June 25, 2010) 